\title{
Recovery characteristics of three anaesthetic techniques for outpatient orthopaedic surgery
}

\author{
G I Randel ${ }^{1}$, S P Kothary ${ }^{2}$, S K Pandit ${ }^{2}$, M Brousseau ${ }^{2}$, L Levy ${ }^{2}$ \\ 'Department of Anesthesiology, Northwestern University, Northwestern Memorial Hospital, \\ Chicago; 2Department of Anesthesiology, University of Michigan Medical Center, Ann Arbor, \\ Michigan, USA
}

The goals of this study are to observe prospectively the perioperative recovery characteristics associated with general anaesthesia (GA), spinal anaesthesia (SAB), and epidural anaesthesia (EPID) in 200 patients scheduled for outpatient knee arthroscopy. Patients were observed from the time they entered the recovery room until they were discharged. Patients were contacted on postoperative days (POD) 1,3 , and 5 . The EPID group had the quickest recovery times $(125 \pm 37$ min, mean \pm SD, ANOVA $P<0.01)$ compared with the GA group (165 \pm 57 min) and $S A B$ group $(167 \pm 51 \mathrm{~min})$. Comparing the side effects of the three anaesthetic techniques, GA was associated with the highest incidence of nausea $(27 \%)$ and vomiting (16\%) on the day of surgery that persisted into the first postoperative day (nausea $41 \%$ and vomiting $22 \%$ ). There was no difference in the incidence of headache overall; however, SAB was associated with a $13 \%$ incidence of postdural puncture (PDP) headache that became apparent on POD 3. All the PDP headaches resolved with conservative therapy by the first postoperative week, except for two patients who required an epidural blood patch. The EPID group followed by the SAB and GA groups, had the highest incidence of backaches on POD 1 (respectively, $63 \%, 41 \%$ and $17 \%$ ). By POD 3 , the incidence of backache was not statistically different between groups. No specific treatment for backache was required. The ideal anaesthetic has not been developed, but our data suggests that an epidural technique is advantageous for knee arthroscopy in terms of a quick recovery and minimal adverse effects.

Key words: Anaesthetic technique, general anaesthesia, regional anaesthesia, spinal epidural, outpatient anaesthesia recovery, postoperative complications, knee arthroscopy

The expansion of outpatient surgery over the past decade has challenged the anaesthesiologist to provide an anaesthetic with quick recovery and minimal adverse effects. Many outpatient surgical procedures are amenable to a variety of anaesthetic techniques. However, side effects are associated with all anaesthetic techniques, such as nausea or vomiting with general anaesthesia and headaches or the inability to void with spinal or epidural anaesthesia. These side effects can prolong the patient's stay in the recovery room or necessitate a return visit to

\section{Accepted: January 1993}

Correspondence and reprint requests 10: Dr Randel, Northwestern University. Northwestern Memorial Hospital, Department of Anesthesiology, 303 E. Superior Street Rm. 360, Chicago, Illinois 60611-3008, USA

Presented in part at the Annual Meeting of the Society for Ambulatory Anesthesia, San Antonio. Texas, April 1989; and at the Annual Meeting of the American Society of Anesthesiologists. New Orleans, Louisiana, October 1989

(4) 1993 Butterworth Hcinemann Ltd $09666532 / 93 / 0100256$ the hospital. Also, the time courses for these common side effects during the perioperative period (when they appear, peak in severity, and resolve) differ for each anaesthetic technique. The pros and cons of each anaesthetic technique must be taken into consideration, as well as the patient's preference and the space and time constraints of the ambulatory surgical centre.

Clarke and Power, comparing postoperative morbidity of spinal anaesthesia with that of general anaesthesia, reported that patients receiving spinal anaesthesia had a high incidence of spinal headaches $(39 \%)$ and backaches $(36 \%)$; they recommended that spinal anaesthesia should not be used in patients under age $40^{\mathrm{t}}$. Epidural anaesthesia has been compared with general anaesthesia for outpatient knee arthroscopy and is reported to offer shorter recovery times than general anaesthesia?2. Finally, a retrospective case review by Orkin reported an increased incidence of severe backaches in patients receiving epidural anaesthesia with chloroprocaine ${ }^{3}$. To date, clinical investigations have not compared, for ambulatory surgery, the recovery characteristics of three common anaesthetic techniques (general 
anaesthesia [GA], subarachnoid block [SAB], and epidural anaesthesia [EPID]) in a single study controlling for the type of operation. The goal of this study was to observe prospectively, in 200 ambulatory patients undergoing knee arthroscopy, the recovery characteristics commonly associated with GA, SAB, and EPID anaesthesia, including the incidence, time course, and severity of adverse effects.

\section{Methods}

This study was approved by our hospital Institutional Review Board. Each patient gave informed consent. Two hundred patients of physical status ASA 1 or 2 scheduled for an arthroscopic procedure of the knee on an ambulatory basis were studied in a non-randomized fashion over a seven-month period. Two surgeons performed all the operations. Three types of anaesthesia were offered (GA, SAB and EPID) to each patient by their anaesthesiologist; the anaesthetic administered was the patient's preference. Local anaesthesia was not offered since a tourniquet was used intraoperatively. Medications, such as fentanyl, midazolam, and droperidol, were administered perioperatively at the discretion of the anaesthesiologists.

\section{General anaesthesia group}

Anaesthesia was induced with thiamylal (4-5 mg kg-1) followed by a short-acting and/or intermediate-acting muscle relaxant. After the trachea was intubated, and at the discretion of the anaesthesiologist, anaesthesia was maintained with a mixture of inhaled agents (nitrous oxide, isofluranc or enfluranc) and fentanyl (1-2 $\mu \mathrm{g} \mathrm{kg}^{-1}$ iv). Propofol was not used for induction or maintenance of anaesthesia. At the end of the procedure, neostigmine and glycopyrrolate were administered to reverse the muscle relaxant effects.

\section{Spinal anaesthesia group and epidural anaesthesia group}

Both regional blocks were performed preoperatively on patients in an induction room adjacent to the operating room to establish a sensory level of at least $T_{8}$. The number of attempts at placement of the needle was recorded for both the spinal and epidural groups. All SABs were performed with a Becton Dickinson 26gauge, Quincke point needle. The spinal needle was placed at the $\mathrm{L}_{2-3}, \mathrm{~L}_{3-4}$, or $\mathrm{L}_{4-5}$ interspace. The local anaesthetic employed was $5 \%$ lidocaine in $7.5 \%$ dextrose plain or with epinephrine, or $1 \%$ tetracaine in $10 \%$ dextrose.

In the EPID group an epidural catheter was placed in all patients with an 18-gauge Touhy-Schliff needle in the $\mathrm{L}_{2,3}$ or $\mathrm{L}_{3-4}$ interspace after localization with $1 \%$ lidocaine. Local anaesthetics employed were either $3 \%$ chloroprocaine or 2\% lidocaine. Epinephrine (1: 200000 solution) and/or fentanyl (1-2 $\mu \mathrm{g} \mathrm{kg} 1$ ) was added to the local anaesthetic at the discretion of the anaesthesiologist.

All recovery times were calculated from the time the patients entered the recovery room until the time they could tolerate oral fluids, ambulate, and void and were discharged home. Symptoms of nausea, vomiting, shivering, lightheadedness, hypotension, and pruritus that occurred on the day of surgery were noted, along with the need for analgesics or antiemetics in the postanaesthesia care unit (PACU) phase. Interviews on postoperative days (POD) 1, 3, and 5 included a standard set of questions regarding the presence and severity of symptoms, such as nausea, vomiting, backache, headache, muscle aches, and pruritus, as well as the need for analgesics. Upon further questioning, the headaches were categorized as 'postdural puncture' (PDP) when the description was consistent with the diagnostic features of PDP headache (onset, location, whether positional in nature, whether relieved in supine position, association with nausea and vomiting) ${ }^{4}$. Data were collected from the anaesthesia records, the PACU records, and from the postoperative interviews. Patients were excluded from entering the study if they would not be available for the postoperative interviews.

\section{Data analysis}

Analysis of variance (ANOVA) with the Scheffé test was utilized to assess differences in recovery times. Fisher's exact tests were used to determine if there were differences in incidences of symptoms on the day of surgery. Patients receiving intraoperative prophylactic antiemetics were excluded from statistical analysis regarding antiemetic treatment in the PACU. A difference was considered significant at a $P$ level $\leqslant 0.05$. Bonferronicorrected Fisher's exact tests (day of surgery) and $\chi^{2}$ (postoperative days 1,3, and 5) were used to compare the anaesthetics pairwise.

\section{Results}

Fourteen of the 200 patients were excluded from statistical analysis because two anaesthetic techniques were employed (GA after regional anaesthesia). Seven of these patients had received a spinal and seven had received an epidural anaesthetic. Thus, the data from 186 patients were analysed; 63 received $G A, 61$ received a $S A B$, and 62 received EPID anaesthesia. Although Surgeon B's surgical time was statistically longer than Surgeon A's $(54.0 \pm 64$ vs $35.0 \pm 13 \mathrm{~min}$, mean $\pm \mathrm{SD}, P \leqslant 0.002$ respectively), all three anaesthetic techniques were equally distributed between them. The demographic data for each treatment group did not differ with respect to gender, weight or height, as shown in Table 1. The mean age of the GA group ( $27 \pm 11 \mathrm{yr}$, mean \pm SD) differed from that of the SAB group $(35 \pm 17 \mathrm{yr}, P=0.003)$. 
Table 1. Demographic data for the groups of patients receiving general, spinal, and epidural anaesthesia

\begin{tabular}{lcccc}
\hline Technique & $\begin{array}{c}\text { Gender } \\
(m / f)\end{array}$ & Age $(\mathrm{yr})$ & $W t(\mathrm{~kg})$ & $H t(\mathrm{~cm})$ \\
\cline { 3 - 6 } & & \multicolumn{4}{c}{$($ mean $\pm \mathrm{sD})$} \\
\hline $\mathrm{GA}$ & $51 / 12$ & $27 \pm 11$ & $79 \pm 15$ & $178 \pm 9.2$ \\
SAB & $48 / 13$ & $35 \pm 17^{*}$ & $79 \pm 15$ & $178 \pm 8.6$ \\
EPID & $41 / 21$ & $30 \pm 12$ & $76 \pm 15$ & $175 \pm 9.2$ \\
\hline
\end{tabular}

* $P=0.003$ when compared to GA by ANOVA with Scheffé correction.

Table 2. Recovery time in minutes (mean $\pm \mathrm{SD}$, and sample size)

\begin{tabular}{lcccc}
\hline Technique & $\begin{array}{c}\text { Oral Intake } \\
(\mathrm{n})\end{array}$ & $\begin{array}{c}\text { Ambulation } \\
(\mathrm{n})\end{array}$ & $\begin{array}{c}\text { Voiding } \\
(\mathrm{n})\end{array}$ & $\begin{array}{c}\text { Discharge } \\
(\mathrm{n})\end{array}$ \\
\hline GA & $99 \pm 45$ & $133 \pm 46$ & $137 \pm 48$ & $165 \pm 57$ \\
& $(59)$ & $(59)$ & $(63)$ & $(63)$ \\
SAB & $80 \pm 34$ & $135 \pm 50$ & $144 \pm 55$ & $167 \pm 51$ \\
& $(60)$ & $(60)$ & $(58)$ & $(61)$ \\
EPID & $71 \pm 34^{*}$ & $99 \pm 28^{\dagger}$ & $108 \pm 36$ & $125 \pm 37^{\dagger}$ \\
& $(62)$ & $(61)$ & $(62)$ & $(62)$ \\
\hline
\end{tabular}

"Time to oral intake differed for EPID vs GA.

'Time to ambulate, void, and discharge differed for EPID vs SAB and GA. The above differences were significant at $P<0.01$ by ANOVA with Scheffé correction.

\section{Recovery times}

The epidural group had significantly shorter recovery times than either the spinal or general group with respect to oral intake, urination, ambulation, and discharge, as shown in Table 2. The patients from the EPID group were discharged 40 minutes earlier than patients from the other groups. Two patients who received a SAB and one patient who had GA required catheterization to relieve urinary retention. Table 3 shows the major component of each anaesthetic technique employed and the discharge time for each technique. Within the SAB group, the type of local anaesthetic used was associated with significantly different discharge times.

\section{Symptoms on the day of surgery}

In the recovery room, the incidence of nausea and vomiting was highest in the GA group and least in the spinal group, as shown in Table 4 . Twenty per cent of patients in the GA group who did not receive intraoperative prophylactic antiemetics required antiemetic therapy in the PACU. This was significantly higher than either the SAB group $(0 \%$ required antiemetic therapy, $P=0.0004)$ or the EPID group $(3 \%$ required antiemetic therapy, $P=0.009$ ). No significant difference was present in the frequency of shivering, lightheadedness, hypotension, or pruritus among the three anaesthetic groups. In the
Table 3. Discharge time for each anaesthetic agent

\begin{tabular}{lll}
\hline Technique ( $\mathrm{n}$ ) & \multicolumn{1}{c}{ Agent ( $\mathrm{n})$} & $\begin{array}{c}\text { Discharge times } \\
(\mathrm{min}) \text { mean } \\
\text { SD }\end{array}$ \\
\hline \multirow{3}{*}{ GA (63) } & isoflurane (39) & $170 \pm 62$ \\
& enflurane (23) & $152 \pm 44$ \\
& isoflurane/enflurane (1) & 250 \\
SAB (61) & lidocaine (44) & $162 \pm 49$ \\
& lidocaine/epinephrine (15) & $170 \pm 49$ \\
& tetracaine (2) & $260 \pm 49^{*}$ \\
& chloroprocaine (14) & $126 \pm 46$ \\
& chloroprocaine/fentanyl (15) & $124 \pm 48$ \\
& chloroprocaine/epinephrine & $125 \pm 23$ \\
EPID (62) & (13) & $130 \pm 52$ \\
& lidocaine (4) & $122 \pm 22$ \\
& lidocaine/fentanyl (14) & 120 \\
& lidocaine/epinephrine (1) & 165 \\
\hline
\end{tabular}

*For the SAB group, tetracaine vs. lidocaine $P=0.008$ and tetracaine vs. lidocaine/epinephrine $P=0.03$.

Table 4. Prevalence of side effects (\%) of the three anaesthetic techniques in the PACU and on postoperative days (POD) 1,3 , and 5

\begin{tabular}{lcccc}
\hline PACU & Nausea & Vomiting & Headache & Backache \\
\hline GA & $27^{\mathrm{a}}$ & $16^{\mathrm{b}}$ & & \\
SAB & 0 & 0 & & \\
EPID & 8 & 5 & & \\
POD 1 & & & & \\
GA & 41 & $22^{\mathrm{d}}$ & 19 & 17 \\
SAB & $10^{\mathrm{c}}$ & 2 & 11 & $41^{\mathrm{e}}$ \\
EPID & 32 & 11 & 18 & $63^{\dagger}$ \\
POD 3 & & & & \\
GA & 8 & 2 & 16 & 19 \\
SAB & 10 & 3 & 19 & 31 \\
EPID & 6 & 0 & 11 & 29 \\
POD 5 & & & & \\
GA & 5 & 2 & 8 & 10 \\
SAB & 10 & 3 & 16 & 20 \\
EPID & 3 & 0 & 12 & 9 \\
\hline
\end{tabular}

anausea in PACU, $P=0.0001$ for GA vs SAB and $P=0.009$ for GA vs EPID.

Vomiting in PACU, $P=0.001$ for GA vs SAB.

-Nausea on POD 1, $P-0.0001$ for SAB vs GA and $P-0.002$ for SAB vs EPID.

dVomiting on POD 1, $P=0.0004$ for GA vs SAB

e. Backache on POD 1, $P=0.003$ for GA vS SAB, $P=0.0001$ for GA vs EPID and $P=0.015$ for SAB vs EPID.

${ }^{\circ}$ One patient in the GA group and four patients in EPID group were lost to follow-up on POD 5.

All significances by $\chi^{2}$ analysis with Bonferroni correction for multiple pairwise comparisons.

EPID group only one patient (who received chloroprocaine with epinephrine) reported pruritus. No patients in any group reported a severe backache. Chi square analysis could not be applied for the analgesic requirements 
because of the various modes of administration (intravenous, oral, and epidural) of opioids and the different types of medications administered to patients (opioid and and nonsteroidal anti-inflammatory agents). There were no unanticipated admissions.

\section{Symptoms on postoperative days 1, 3, and 5}

Table 4 displays the overall prevalence of side effects on postoperative days 1, 3, and 5 for the three anaesthetic groups. No statistically significant differences were present in the frequency of muscle aches, pruritus, or analgesic requirements between the groups. On POD 1, the incidence of nausea, vomiting and headache was greatest in the GA group while backaches occurred more frequently in the two regional anaesthesia groups. Of the $41 \%$ of patients in the GA group with nausea on POD 1 , $69 \%$ rated it as mild, $23 \%$ moderate and $8 \%$ severe. By POD 3 there were no statistically significant differences between groups.

Headache occurred with the same frequency on POD I in all the anesthetic groups. By POD 3, the frequency as well as the severity of the headaches was more pronounced in the spinal group; however, no significant differences existed. Although $12(19 \%)$ patients in the SAB group reported a headache on POD 3, only eight of these patients $(13 \%)$ had symptoms consistent with a PDP headache as previously described. Seven out of the eight PDP headaches occurred in patients 33 years of age or younger. The PDP headache was rated as severe in five patients, moderate in one patient and mild in two patients. Two patients required treatment with an epidural blood patch. No wet taps were reported by the anaesthesiologist in the EPID group and no PDP headaches occurred in this group.

Backaches were present in all the groups, with the EPID group having the highest incidence on POD 1 $(63 \%)$ followed by the SAB group $(41 \%)$ and GA group $(17 \%)$. In terms of severity, patients in the EPID group with a backache on POD 1 rated it as mild, $42 \%$; moderate, $52 \%$ and severe, $6 \%$ compared to mild, $60 \%$; moderate, $32 \%$ and severe, $8 \%$ in the SAB group and mild, $73 \%$; moderate, $27 \%$ and severe, $0 \%$ in the GA group (not statistically different).

Within the EPID group, data from patients receiving chloroprocaine, chloroprocaine with epinephrine and chloroprocaine/fentanyl were pooled into a chloroprocaine-based group and data from patients receiving lidocaine, lidocaine with epinephrine and lidocaine/fentanyl were pooled into a lidocaine-based group and the incidence and severity of backache compared. The mean ( \pm SD) total volume of local anaesthetic given was $32.9 \pm 9.8 \mathrm{ml}$ for the chloroprocaine group and $31.9 \pm 9.8 \mathrm{ml}$ for the lidocaine group. Sixty-four per cent of patients in the chloroprocaine-based group reported a backache on POD 1 compared to $58 \%$ in the lidocainebased group. Severity of backache was mild, $30 \%$; moderate, $59 \%$ and severe, $11 \%$ in the chloroprocainebased group versus mild, 64\%; moderate, $27 \%$ and severe, $9 \%$ in the lidocaine-based group. None of these differences were statistically significant.

By POD 3, there was no difference in the incidence of backaches between the EPID, SAB or GA groups. No specific treatment for backache was required in any of the groups.

\section{Discussion}

Our current criteria for discharge home after an outpatient surgical procedure include the ability to tolerate oral fluids, ambulate, and void. The patients in the EPID group met these criteria significantly sooner than the GA and SAB group. We propose two reasons for this result. First, the EPID group received a less dense motor block than did the SAB group, and consequently could void and ambulate significantly sooner. Secondly, the EPID group had less nausea than the $\mathrm{G} \Lambda$ group which reduced the need for postoperative antiemetic treatment with its sedative side effects. This corroborates previous investigators' findings of longer discharge times in the recovery room in outpatients who suffer from postoperative nausea and vomiting. Interestingly, the high incidence of nausea and vomiting in the GA group continued (and even increased slightly) into the first postoperative day; this persistence may be attributed, in part, to the use of narcotic analgesics in conjunction with inhalation agents. Although not included in this study, the routine administration of prophylactic antiemetics or use of newer intravenous agents (propofol) may decrease the incidence of nausea and vomiting ${ }^{5}$.

At our institution, GA and EPID anaesthesia is employed more often than SAB in young ambulatory patients to avoid the possibility of PDP headaches. The incidence of PDP headache after subarachnoid block in our patient population remains unacceptably high at $13 \%$, even with use of a 26-gauge Quincke point needle. This was lower than the $18 \%$ incidence reported by Clarke and Power, however, we concur with their recommendation that spinal anaesthesia should not be used in ambulatory patients under age $40^{\prime}$. Some authors report further decreases in the incidence of PDP headaches by utilizing a smaller gauge or different variety of spinal

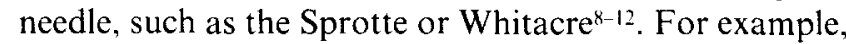
Dahl and coauthors, utilizing a 29-gauge needle for SAB in patients undergoing knee arthroscopy, found a $11 \%$ incidence of PDP headache ${ }^{8}$.

Continuing postoperative follow-up was key in our ability to detect PDP headache. Our PDP headaches were not apparent until POD 3, perhaps because these orthopaedic patients are instructed to elevate their operative leg for the first day or two postoperatively and remain in a recumbent position. We believe therefore that follow-up on patients receiving a SAB for an arthroscopic procedure of the knee should be extended to at least POD 3 or 5. Often, these patients did not associate their headaches with the regional anaesthetic administered days before.

Previous studies have reported a $19-30 \%$ incidence of 
backaches following spinal or general anaesthesia for different types of surgical procedures ${ }^{13.14}$. Differences in the incidence of backache could not be accounted for by patient position (supine, lithotomy, prone, lateral or sitting) during surgery or anaesthetic technique, however the incidence did increase with prolonged surgical duration. Dahl compared SAB with GA in patients undergoing arthroscopy of the knee and found an incidence of backaches of $26 \%$ in the SAB group versus $4 \%$ in the GA group; all of the backaches in his SAB group were rated as 'light' in severity". Surgical position was not described. The incidence of backaches in our study was much higher, ranging from $17 \%$ in the GA group to $63 \%$ in the EPID group. The surgical position used at our institution may account for part of this result since the SAB and GA groups also showed a high incidence of backaches. In our study, only onc surgical position was employed. The patient was positioned supine on the operating table with the nonoperative leg flexed at the knee with the hip in a neutral to slightly extended position; and the operative leg flexed at the hip while the knee was torqued at different angles during the procedure.

An increased incidence of severe back pain has been noted by several investigators in retrospective reviews when $3 \%$ chloroprocaine was used in the epidural space ${ }^{3.15}$. It has been suggested that this back pain is related to the administration of a volume greater than 25 $\mathrm{ml}$. The average volume of local anaesthetic utilized in our study was $32.6 \pm 9.6 \mathrm{ml}$. In contrast to Orkin's finding that all patients given over $50 \mathrm{ml}$ of $3 \%$ chloroprocaine had back pain, one patient in our study received $56 \mathrm{ml}$ of chloroprocaine with fentanyl but voiced no complaints of back pain. While this study was not designed (not randomized or double blind) to compare chloroprocaine with lidocaine administered in the epidural space, data from the EPID group was pooled into lidocaine-based and chloroprocaine-based anaesthetics. The incidence and severity of backache was not found to be different, which concurs with a previously published prospective, randomized, double-blind study ${ }^{16}$. The backaches present in our EPID group were not associated with any neurologic signs or deficits and did not require treatment.

This prospective study was not randomized. Randomization was precluded by an ethical belief that full informed consent, including discussion of all possible anaesthetic techniques needs to be given to patients prior to their procedure. Obviously this may introduce certain biases, however, the only demographic difference between our patient groups was in the older age of the SAB group. This probably reflects the general consensus regarding an increased incidence of $P D P$ headache in a younger population. Also, this study did not encompass patients' prior history of headaches or backaches. Because the patient was allowed to choose their anaesthetic technique, patients with previous back problems may be underrepresented in the SAB and EPID groups. Finally, no attempt was made to limit the medications employed perioperatively. The intent of this study was to describe the outcome of three anaesthetic techniques and their short- and long-term recovery characteristics in the context of our daily anaesthetic practice.

\section{Conclusion}

The ideal anaesthetic has not been developed, but our data suggest that an epidural technique is advantageous for knee arthroscopy. Epidural anaesthesia provided the shortest recovery times with minimal adverse effects. The backaches present in the EPID group required no treatment and were not associated with neurologic sequelae. Although PDP headache can occur with epidural anaesthesia, the incidence is unlikely to be as high as we observed in the SAB group (13\%). Nausea and vomiting in the EPID group was limited and did not affect discharge times.

\section{Acknowledgements}

Grateful acknowledgement is respectfully owed to Dr A Vanderspek, Associate Professor of Anesthesiology, Dr J Wahr, Instructor of Anesthesiology, for their helpful comments and Dr M Schork, Professor of Biostatistics, for his statistical guidance.

\section{References}

1 Clarke GA, Power KJ. Spinal anaesthesia for day case surgery. Ann R Coll Surg Eng/ 1988; 70; 144-6

2 Parnass SM, McCarthy RJ, Bach B, et al. A prospective evaluation of epidural versus general anesthesia for outpatient arthroscopy (abstract). Anesthesiology' 1990; 73 A23

3 Orkin FK, Bogetz MS. Back pain following uncomplicated epidural anesthesia with chloroprocaine (abstract). Anesthesiology 1989; 71: A716

4 Bridenbaugh PO, Greene NM. Spinal (subarachnoid) neural blockade. In Cousins MJ, Bridenbaugh PO (eds) Neural blockade in clinical anesthesia and management of pain. Philadelphia, Lippincott, 1988; 246

5 Pandit SK, Kothary SP, Pandit VA, Randel G. Levy L Dose-response study of droperidol and metoclopramide as antiemetics for outpatient anesthesia. Anesth Analg 1989; 68: 798-802

6 Johnston R, Noseworthy T, Anderson B, Konopad E, Grace M. Propofol versus thiopental for outpatient anesthesia, Anesthesiology 1987; 67: 431-3

7 Doze VA, Shafer A, White P. Propofol-nitrous oxide versus thiopental-isoflurane-nitrous oxide for general anesthesia. Anesthesiology 1988; 69: 63-71

8 Dahl JB, Schultz, P, Anker-Moller E, Christensen EF, Staunstrup HG, Carlsson P. Spinal anaesthesia in young patients using a 29-gauge needle: Technical considerations and an evaluation of postoperative complaints compared with general anaesthesia. Br J Anaesth 1990; 64: 178-82

9 Sears DH, Leeman MI, O'Donnell RH, Kelleher JF, Santos GC. Incidence of postdural puncture headache in cesarean section patients using the $24 \mathrm{G}$ Sprotte needle (abstract). Anesthesiology 1990: 73: A 1003

10 Snyder GE, Person DL, Flor CE and Wilden RT. 
Headache in obstetrical patients; comparison of Whitacre needle versus Quincke needle (abstract). Anesthesiology 1990; 71: A860

11 Flaaten H, Rodt DA, Vamnes J, Rosland J, Wisborg T, Koller ME. Postdural puncture headache: A comparison between 26- and 29-gauge needles in young patients. Anaesthesia 1989; 44: 147-9

12 Gielen M. Post dural puncture headache (PDPH): A Review. Regional Anesthesia 1989; 14: 101-6

13 Brown EM, Elman DS. Postoperative backache. Anesth Analg 1961; 40: 683-5
14 Pitkanen M, Rosenberg PH. Minor complications following anaesthesia in young adults for orthopaedic surgery of the lower extremity. Ann Chir Gynaecol 1987; 76: $99-103$

15 Hynson, JM, Sessler DI, Glosten B, McGuire J. Back pain following chloroprocaine epidural anesthesia (abstract). Anesthesiology 1990; 73: A48,

16 Levy L, Randel GI, Pandit SK. Does chloroprocaine (Nesacaine MPF) for epidural anesthesia increase the incidence of backache? (letter to the editor) Anesthesiology 1989; $71: 476$

\section{Second European Congress on} Ambulatory Surgery

1 Day Surgery in Europe

Brussels, Belgium

\section{9-20 March 1993}

The congress will aim to reflect the dynamic growth of interest in ambulatory surgery witnessed throughout Europe and the Rest of the World. Plenary sessions will examine the structural, organizational and financial aspects of ambulatory surgery, and be supported by sessions dedicated to Surgery and Anaesthesia. A comprehensive series of poster presentations has also been organized.

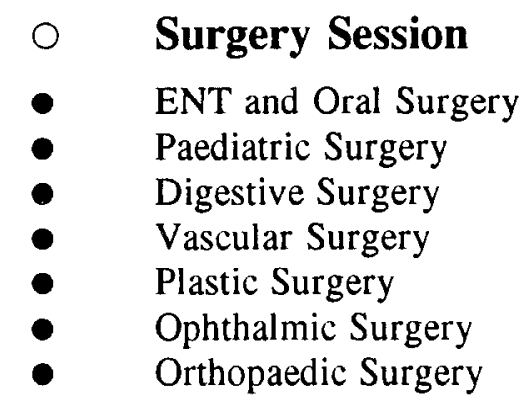

\section{$\bigcirc \quad$ Anaesthesia Session}

- Which Anaesthetic?

- Extremes of Age \& Anaesthesia

- Scientific Papers on Anaesthesia

- Controversies in Anaesthesia

For late registration please contact the Administrative Secretariat: Medicongress, Waalpoel 28, B-9960 Assenede, Belgium. Tel: +32 (0)91 444096 Fax: +32 (0)91 444010 